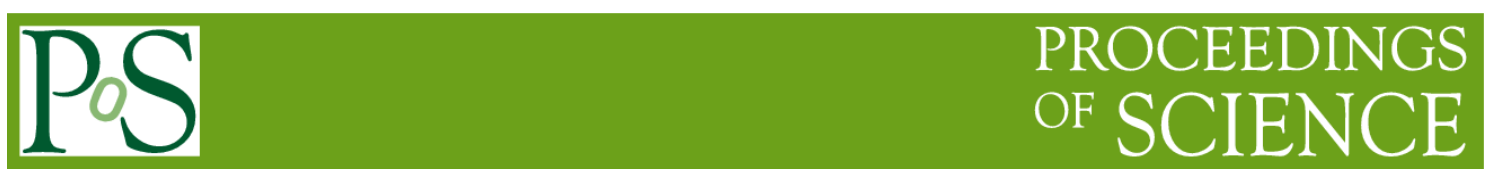

\title{
More results from the OPERA experiment
}

\section{Yury Gornushkin ${ }^{1}$ on behalf of the OPERA Collaboration}

Joint Institute for Nuclear Research

6, Joliot-Curie str., Dubna, 141980, Russia

E-mail: gornushk@yandex.ru

The OPERA experiment has recently assessed the discovery of nu-tau neutrinos in the CNGS beam with a significance larger than 5 sigma, thus successfully fulfilled its main goal. The ongoing activity is aimed at the completion of the data analysis related to other topics. Updated results presented including the search for $v_{\mu} \rightarrow v_{\mathrm{e}}$ oscillations.

38th International Conference on High Energy Physics

3-10 August 2016

Chicago, USA

\footnotetext{
${ }^{1}$ Speaker
} 


\section{Introduction}

The OPERA experiment was designed in late 90's to prove unambiguously the oscillation of muon neutrinos to tau neutrinos in atmospheric sector by direct detection of tau neutrinos in the muon neutrino beam from CERN at a distance of $730 \mathrm{~km}$ from the beam origin. Although the neutrino oscillations phenomenon, proposed in the 50's by B.Pontecorvo, could naturally explain the deficit of neutrino flavours observed in cosmic ray showers and in the neutrino flux from the Sun, for many years this possibility was considered as too exotic, and some physicists were skeptical about the experiments observing such deficit. The physics community finally changed its mind when in 1998 the SuperKamiokande experiment presented convincing results on the dependence of the atmospheric neutrinos flux on the distance between the detector and the neutrino production region. The most probable explanation of the observed dependence was the change of the flavour of neutrinos from muon to tau on their way to the detector. But a direct observation was still missing. Indeed tau neutrinos are very difficult for registration and had not been detected yet by that time. The OPERA Collaboration set out to perform a challenging search for tau neutrinos in the originally pure muon neutrino beam from the CERN accelerator with an apparatus located at the Gran Sasso underground Lab.

\section{OPERA experimental technique.}

OPERA exploited the so-called ECC technique (Emulsion Cloud Chambers) to detect tau neutrinos. The target part of the OPERA hybrid detector (Fig.1) consisted of 150000 elementary units (bricks) made of 56 thin layers $(1 \mathrm{~mm})$ of lead interleaved with 57 nuclear emulsion films where the short tracks of tau lepton could be reconstructed with micrometer accuracy thanks to the excellent emulsion spatial resolution. The target bricks were arranged in 62 target walls (with a total mass of $1250 \mathrm{t}$ ) interleaved with tracking electronic detectors (Target Tracker, TT) which provided real time event information and served for the prediction of the bricks where the neutrino interaction most probably had occurred. The OPERA detector had also two magnetic spectrometers for the identification of muons produced in the neutrino interactions and for their momentum measurement. Once the brick with the neutrino interaction was selected, it was extracted from the detector by a dedicated robot. The choice of the brick made by TT was first confirmed by a separate emulsion dublet attached to each brick (CS). In case of presence of the tracks corresponding to the signals in the TT, the brick was dismounted, the emulsion films were developed and scanned with help of fast automatic microscopes developed ad hoc by the Collaboration. By emulsion scanning, 3D coordinates of the silver grains which mark in the emulsion the traces of charged particles with submicronic precision, are recorded. The tracks are reconstructed by standard algorithms as well as the neutrino interaction 
vertexes. Dedicated procedures were applied to the tracks in order to find a large kink along it or a large impact parameter with respect to the primary vertex, both as a signature of short lived particle decay. When a secondary (decay) vertex is found, a full kinematical analysis is performed: the momentum of the charged particles is measured via their multiple scattering in the brick lead layers and the event energy measured using information from the electronic detectors.

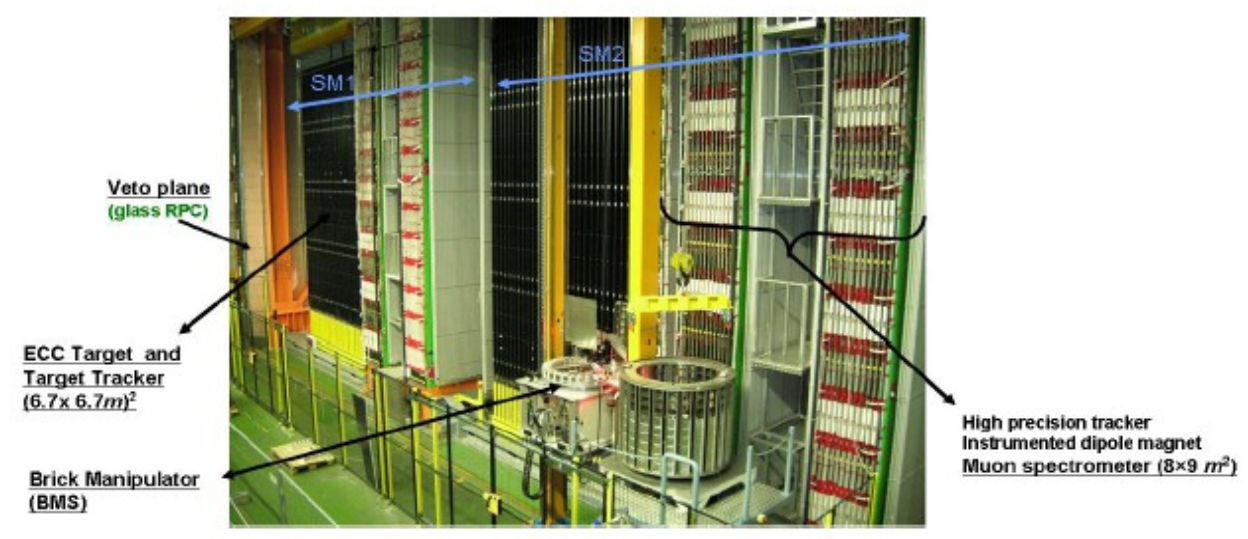

Figure 1. The OPERA detector.

\section{OPERA data taking and analysis.}

The OPERA electronic detectors were commissioned in 2006. In the same year the CNGS neutrino beam from CERN was also commissioned. In 2007-2008 the production of 150000 target bricks and detector filling have been completed. The CNGS beam which met technical problems in 2007 became available for the physics run in 2008. During five years of data taking (2008-2012), $17.97 \times 10^{19}$ of protons were delivered on CNGS target, and 19505 neutrino interaction were registered by the OPERA detector.

The neutrino event analysis was performed for all neutral current (NC)-like events and for CC-like events with a muon momentum below $15 \mathrm{GeV} / \mathrm{c}$ to reduce the background. The scanning and the analysis of the 1 st and 2 nd most probable bricks were performed and $5 v_{\tau}$ candidates were observed. The expected signal and background for the different $\tau$-decay channels are reported in Table 1 . In the oscillation analysis the observed number of $v_{\tau}$ candidates for each channel was considered as an independent Poisson process with individual signal and background, and the significance of the observed $v_{\tau}$ candidates was evaluated as the probability that the background can produce a fluctuation greater than or equal to 5 events (Table 1). As all possible background sources (decays of charmed particles, particle hadronic reinteractions, and large angle scattering of muons) were estimated to contribute about of 0.25 events, tau neutrino appearance was proved with a significance of 5.1 standard deviations [1]. Thus, the mission of the OPERA experiment was successfully completed. 
Table 1. Summary of observed tau neutrino events and expected backgrounds.

\begin{tabular}{|c|c|c|c|c|c|c|}
\hline \multirow[b]{2}{*}{$\begin{array}{l}\text { Decay } \\
\text { channel }\end{array}$} & \multicolumn{4}{|c|}{ Expected background } & \multirow{2}{*}{$\begin{array}{c}\text { expected signal } \\
\text { events } \\
\Delta m 2= \\
2.44 \times 10-3 \mathrm{eV} 2\end{array}$} & \multirow[b]{2}{*}{$\begin{array}{c}\text { Observed } \\
\text { events }\end{array}$} \\
\hline & Charm & $\begin{array}{l}\text { Had. Re- } \\
\text { interaction }\end{array}$ & $\begin{array}{l}\text { Large mиоn } \\
\text { scattering }\end{array}$ & Total & & \\
\hline$\tau \rightarrow 1 h$ & $0.017 \pm 0.003$ & $0.022 \pm 0.006$ & - & $0.04 \pm 0.01$ & $0.52 \pm 0.10$ & 3 \\
\hline$\tau \rightarrow 3 h$ & $0.17 \pm 0.03$ & $0.003 \pm 0.001$ & - & $0.17 \pm 0.03$ & $0.73 \pm 0.14$ & 1 \\
\hline$\tau \rightarrow \mu$ & $0.004 \pm 0.001$ & - & $0.0002 \pm 0.0001$ & $0.004 \pm 0.001$ & $0.61 \pm 0.12$ & 1 \\
\hline$\tau \rightarrow e$ & $0.03 \pm 0.01$ & - & - & $0.03 \pm 0.01$ & $0.78 \pm 0.16$ & 0 \\
\hline Total & $0.22 \pm 0.04$ & $0.02 \pm 0.01$ & $0.0002 \pm 0.0001$ & $0.25 \pm 0.05$ & $2.64 \pm 0.53$ & 5 \\
\hline
\end{tabular}

The OPERA Collaboration is continuing the data analysis to fully exploit the physics potential of the registered neutrino events. In particular, OPERA published the results on the sterile neutrino search in the $v_{\mu} \rightarrow v_{\tau}$ oscillation analysis [2] as well as the results of the $v_{\mu} \rightarrow v_{\mathrm{e}}$ oscillations analysis using data collected in 2008-2009 [3]. Now OPERA is ready to present the results of the oscillation analysis related to the full statistics of 2008-2012 and based on advanced approach [4]. Other ongoing searches concern the $v_{\mu}$ disappearance analysis, the search for $v_{\tau}$ with less stringent selection requirements, and the study of the seasonal variation of the cosmic muon flux at LNGS.

\section{References}

[1] N.Agafonova et al., Discovery of tau neutrino appearance in the CNGS neutrino beam with the OPERA Experiment, Phys.Rev.Lett. 115 (2015) no.12, 121802 [hep-ex/1507.01417]

[2] N.Agafonova et al., Limits on muon-neutrino to tau neutrino oscillations induced by a sterile neutrino state obtained by OPERA at the CNGS beam, JHEP 1506 (2015) 069 [hep-ex/1503.01876]

[3] N.Agafonova et al., Search for muon-neutrino to electron-neutrino oscillations with the OPERA experiment in the CNGS beam, JHEP 1307 (2013) 004 [ hepex/1303.3953]

[4] A.Palazzo, Consistant analysis of the $v_{\mu} \rightarrow v_{\mathrm{e}}$ sterile neutrinos searches of ICARUS and OPERA, Phys.Rev. D91 (2015) no.9, 091301 [hep$\mathrm{ph} / 1503.03966]$ 